\title{
Challenges in Cancer Care of Elderly
}

\author{
Virender Suhag1 ${ }^{*}$, B. S. Sunita², Arti Sarin ${ }^{3}$, A. K. Singh ${ }^{4}$ \\ ${ }^{1}$ Department of Radiation Oncology, Army Hospital (Research \& Referral), Delhi, India \\ ${ }^{2}$ MD DNB Pathology, Base Hospital, Delhi, India \\ ${ }^{3} \mathrm{MD}$ (Radiodiagnosis), DNB Radiation Oncology, Army Hospital (Research \& Referral), Delhi, India \\ ${ }^{4} \mathrm{MD}$ Radio-Diagnosis and Trained in Radiation Oncology, Department of Radiodiagnosis, Army Hospital \\ (Research \& Referral), Delhi, India \\ Email: *virendersuhag@gmail.com
}

Received 24 December 2014; accepted 11 January 2015; published 19 January 2015

Academic Editor: Maria Chan, Memorial Sloan-Kettering Cancer Center at Basking Ridge, USA

Copyright (C) 2015 by authors and Scientific Research Publishing Inc.

This work is licensed under the Creative Commons Attribution International License (CC BY).

http://creativecommons.org/licenses/by/4.0/

c) (i) Open Access

\begin{abstract}
As a result of vast global improvement of health care and living conditions, the world population is aging. In developed countries, more than half of the cancers occur in patients aged 70 and older. In booming Asian nations, such as India, the aging trend is particularly striking, and therefore geriatric oncology is rapidly coming at the foreground of oncology practice. As these patients have special needs and a different approach to treatment, there is a strong need for the emergence of geriatric oncology as a sub specialty in oncology. Scientific data show that a geriatric assessment identifies many problems in older people with cancer, adds prognostic information, and might improve the outcomes of these patients. There is a genuine unmet requirement to design and implement the following: development of individually tailored geriatric assessment tools for different oncology centers; cooperation of aging and cancer research in the understanding of cancer biology, aging and physiology; improved clinical study designs; development of geriatric oncology programs; and screening tools for geriatric patients made accessible to family physicians.
\end{abstract}

\section{Keywords}

Geriatric Oncology, Geriatric Assessment, Aging, Cancer

\section{Introduction}

Due to improvement in public health, now more individuals are living longer and the proportion of those living

${ }^{*}$ Corresponding author.

How to cite this paper: Suhag, V., Sunita, B.S., Sarin, A. and Singh, A.K. (2015) Challenges in Cancer Care of Elderly. International Journal of Medical Physics, Clinical Engineering and Radiation Oncology, 4, 25-31.

http://dx.doi.org/10.4236/ijmpcero.2015.41004 
beyond 60 years has increased significantly and will increase further over the next 20 years. It is projected that by 2020 , the population of elderly globally would be more than 700 million with two-third belonging to the developing countries [1] [2]. Advancing age is a high risk factor for cancer, with persons over 65 accounting for $60 \%$ of newly diagnosed malignancies and $70 \%$ of all cancer deaths, mainly prostate, bladder, colon, uterus, pancreas, stomach, rectum, and lung carcinomas [1] [3] [4]. Most countries are experiencing an increase in older individuals with cancer, both in terms of absolute numbers and as a proportion of the total population. The incidence of cancer in those over 65 is 10 times greater than in those younger than 65 and the cancer death rate is 16 times greater in patients over 65 compared to younger patients [3] [5].

This graying of the population and increasing prevalence of cancer amongst elderly will have important dermographic and socioeconomic consequences. It will profoundly impact the health care industry as more resources and personnel will be required to take care for the elderly. Moreover, this group is less extensively investigated and probably receives less appropriate treatment than younger patients. Usually, the elderly cancer patients are considered not suitable for a curative treatment approach. The choice of treatment modalities in term of radiotherapy, chemotherapy, or even surgery becomes a difficult decision both for the doctor and patients and their relatives. More often elderly patients are put on conservative or less aggressive treatments. Thus, a large proportion of patients are denied the benefit of aggressive treatment. Moreover, many of the prospective clinical trials have excluded this group of patients from the trials, and thus, no worthwhile clinical studies were forthcoming from this age group, especially in developing countries [6] [7]. Therefore, there seems to be a need to integrate and optimize treatment planning for elderly cancer patients to achieve an improved tumor control, quality of life, and survival with better health care [5] [8]-[10]. There is an important role for education of healthcare providers, the elderly and their caregivers to eliminate age bias, institute best practices for screening and prevention and achieve optimal care for older patients with cancer [11]-[16]. The aim of this article is to highlight issues unique to the older adult population and to propose a roadmap for care of such cancer patients.

\section{Physiology of Aging}

Some of the reasons why cancer is more common in the elderly include telomere shortening and defective deoxyribo nucleic acid (DNA) repair mechanisms, age-related alterations in the immune system, accumulation of random genetic mutations leading to oncogene activation, amplification or decreased tumor suppressor gene activity, life-time carcinogen exposure, and hormonal alterations or exposure. For the proper management of these patients, a better understanding of the physiology of aging is required. In geriatric age group, there is increase in the body fat and decreased total body water and muscle mass secondary to hormone dysfunction and decreased protein synthesis, loss of sweat glands, decreased gastrointestinal motility and absorption, and decreased hepatic and renal functions. There is decrease in weight of the brain and also there is a decline in the amount of neurotransmitters with age. There is also immunologic dysregulation and compromised marrow reserve. Aging is associated with a progressive decline in the functional reserve of multiple organ systems. This may influence pharmacokinetics and pharmacodynamics of antineoplastic drugs and reduce the tolerance of normal tissues for treatment complications. Age may be construed as a progressive loss of stress tolerance, due to decline in functional reserve of multiple organ systems, high prevalence of comorbid conditions, limited socioeconomic support, reduced cognition, and higher prevalence of depression. Aging is highly individualized; chronologic age may not reflect the functional reserve and life expectancy of an individual [5] [17]-[19].

\section{Challenges Posed by Multiple Comorbidities}

Older people are more likely to have comorbidities and geriatric syndromes such as incontinence, falls, functional decline, polypharmacy and delirium [20] [21]. The strategies to prevent, screen or treat cancer in older individuals will potentially be impacted by these comorbidities. Physical, cognitive, or emotional issues add to the complexity of their care needs. The typical goals of cancer care are influenced by the confluence of health and social issues experienced by older adults, with an emphasis on quality of life playing a critical role in determining which treatments they are willing to accept. Available evidence suggests that the majority of chronically ill older adults value improving or maintaining function more than prolonging life. Quality in cancer care mandates partnering with older adults to enable them to anticipate changing health needs, incorporate their preferences into plans of care, and consistently act based on their individual goals. Ensuring that high-quality services such as palliative care or hospice are available in a timely manner also is essential to addressing this challenge. 
Because many older adults face myriad health issues, care is often provided by a diverse and fragmented group of clinicians. Substantial adjustments in the current model of care delivery are required to ensure that the care of older adults with cancer is well coordinated and comprehensive, while also focusing on individuals' goals and preferences [5] [20]-[24].

\section{Factors Affecting Cancer Treatment Decisions}

Decisions about cancer treatment in the older person should be made in the same way that decisions about cancer treatment are made for younger adults and should not focus on the person's age alone. Treatment discussions and decisions, made between the health care team and the older adult with cancer and their family, should be based on but not limited to the following factors [8] [13] [17] [22] [24] [25]:

- The type of cancer and its stage

- Available treatment options

- The risks and benefits of each treatment option

- The person's assessment of his or her goals of treatment and tolerance of risk, including side effects

- The presence of medical conditions other than cancer that may put the older adult with cancer at an increased risk for treatment-related side effects or complications

- The effect of intensive treatment on the physical, emotional, and social well-being of the older adult

- The person's concept of quality of life, their perceptions and decisions may differ from those of family members, friends, and caregivers.

- Emotional and social limitations, including the level of caregiver support and feelings of social connection or isolation for people with cancer who are living alone

- Financial limitations, as older adults are more likely to have limited resources and live on a fixed income, which may cause them to refuse procedures or treatment due to cost.

- Spiritual beliefs, as many older adults have already come to terms with death and dying due to chronic illnesses, the loss of a spouse, or advanced age.

\section{Comprehensive Geriatric Assessment (CGA)}

The assessment of these patients requires a different approach and comprehensive geriatric assessment (CGA) is recommended for older patients with cancer, which best accounts for the diversities in the geriatric population. The CGA may be defined as a multidisciplinary evaluation in which the multiple problems of older persons are uncovered, described, and explained, if possible, and in which the resources and strengths of the person are catalogued, need for services assessed, and a coordinated care plan developed. This adds information to the presently used measures of functional status in oncology, such as the Karnofsky or Eastern Cooperative Oncology Group Performance Status. The CGA has been designed by geriatricians as a multidimensional assessment of general health status based on validated geriatric scales and tests that produce an inventory of health problems, allowing the development of an individualized geriatric intervention program [5] [10] [17] [26]-[28].

The core components of the CGA are functional status, cognition, mood and emotional status, social support, financial concerns, nutritional status, comorbidities and polypharmacy, geriatric syndromes (fall risk, confusion, urinary incontinence, visual or hearing impairments), goals of care, and advance care planning. The content of the assessment varies with the care setting (e.g., home, clinic, hospital, or nursing home). In many settings, the CGA process relies on a core team consisting of a physician, a nurse, and a social worker, who obtain assistance as needed from other health care professionals (e.g., nutritionist, physical therapist, and/or psychologist). The advantages of the CGA include: 1) Recognition of potentially treatable conditions such as depression or malnutrition, which may lessen the tolerance of cancer treatment and be reversed with proper intervention; 2) Assessment of individual functional reserve; 3) Gross estimate of individual life expectancy; 4) Adoption of a common language to classify older cancer patients. Limitations to the widespread use of the CGA in everyday practice are the considerable time and human resources needed to conduct the assessment and the failure of some health insurance systems to reimburse it [5] [13] [29]-[31].

\section{Lack of Clinical Data in Gertiatric Patients}

Responses to cancer diagnoses and treatments are influenced by the aging process. To provide quality cancer care for older adults, research in this population needs to be enhanced. Although the majority of patients with 
cancer and cancer survivors are older adults, historically they have been and continue to be underrepresented in all types of cancer trials [7] [32]. This may be because many older patients present in advanced stage and have poor physical condition. It may also be due to reluctance on the part of the physicians or patients to enroll in adjuvant trials. Therefore, fewer data are available for assessing the risks and benefits of cancer treatments that carry the possibility of adverse effects and functional decline. Furthermore, chronological age does not automatically equate with functional age. For health care professionals to extrapolate study results to patients seen in general practice, a more detailed characterization of the study population is needed, such as could be captured by a geriatric assessment that provides measures of functional rather than chronological age. This would expand the breadth and depth of data available for understanding the characteristics of patients who may benefit from treatment as well as for developing interventions for those vulnerable to adverse effects. For patients and physicians to adequately balance the risks and benefits of therapy, it is recommended that the research studies include a plan to recruit a population that represents the age distribution and health-risk profiles of patients with cancer [6] [33]-[37].

\section{Need of Dedicated Geriatric Care Providing Team}

Although the number of patients with cancer has increased and the majority of these individuals are older adults, there is a professional workforce shortage of oncologists, geriatricians, and members of the multidisciplinary care team who have expertise in geriatrics. Every country should have a national workforce commission to plan for the challenges of an aging population and the complexity of care required by older adults with cancer [8] [15] [38]. A workforce needs to be developed that understands and values the contributions of the multidisciplinary team and has the knowledge of geriatrics principles essential to support older adults coping with cancer. Therefore, training in geriatrics principles should be included among the core competencies of all individuals caring for older adults [39] [40].

The increase in the number of older adults, the association of cancer with aging, the workforce shortage, and the financial stressors across the health care system and family networks all contribute to a crisis in cancer care that is most pronounced in the older population. Critically appraising the health care system and redefining these challenges as opportunities would help begin charting a new and better course of action that will improve the quality of cancer care for older adults with cancer in the decades to come. Every geriatric oncology system should develop a roadmap to achieve an integrated system that recognizes that cancer care must be viewed within the context of aging. Indeed, addressing the unique needs of an aging population is central to improving the quality of cancer care [15] [16].

\section{Support and Training of Caregivers}

The association between cancer and aging will substantially affect health care systems and health care economics, with an adverse effect on those family members regarded as "family caregivers." Most "hands on" care is provided by family and friends who play a key role in the health care infrastructure, providing support and care for older adults with cancer, often with little or no training. The physical, psychological, financial, and emotional tolls borne by caregivers are gradually being recognized; however, few programs prepare and support the caregivers of older adults with cancer. Effective ways to train and support caregivers must be developed. The only way we can develop adequate care for such a huge number of patients is to use a "train the trainer" approach. There is a need to develop a cadre of geriatric oncology specialists, committed to research and teaching in this particular field. Geriatric oncology specialists will train their geriatrics and oncology colleagues, who in turn will form trained teams in their institutions. These local institution-based teams will train the private practice colleagues in their area. Institutions and private practices will need to develop multidisciplinary structures to treat what already is and increasingly will be a major part of their patients. It is estimated right now that in tertiary centers half of the older cancer patients are in need of a comprehensive oncogeriatric approach. These structures will need to include not only oncologists and geriatricians, but also a team of social workers, dieticians, physical therapists, pharmacists, nurses and mid-level practitioners with knowledge pertaining to this patient population [38] [39] [41].

\section{Recommendations}

- Cancer treatment recommendations and assumptions about elderly patients should not be based solely on 
their date of birth. Early and appropriate assessments should be used to indicate who will tolerate what treatment. The assessment should not only inform a dialogue about cancer treatment, but should identify and address unmet physical, psychological and social support needs [8] [17].

- Patients' other health problems and existing medications should be better addressed to ensure they get the maximum benefit from their cancer treatment, ideally with guidance from an elderly care physician.

- Patients should be provided with information about local services which can offer practical and social support during cancer treatment. An older person should never refuse treatment because they are having difficulties with transport or caring for a relative [13] [15].

- More elderly people should have the opportunity to enroll on clinical trials; other forms of evidence need to be gathered to build a solid evidence base.

- More research should be carried out to understand the extent of under treatment and the factors contributing to it.

- More elderly care specialists should be engaged as an active part of the cancer care team and adopt a multidisciplinary approach to the assessment and management of all patients [13] [15] [42].

- Establish services and clear referral pathways for both outpatients and inpatients to address needs identified by assessment. This includes establishing clear links with voluntary sector agencies, social services, and specialist teams such as falls prevention teams, continence specialists and dementia specialists.

- Ensure that effective communication systems are in place to facilitate coordinated care and informed decision making.

- Ensure that all clinical and non-clinical staff is supported with the training and access to resources required to conduct appropriate assessment and follow up care of all patients [8] [17] [42].

\section{Conclusion}

In summary, the number of older people with cancer is expected to significantly increase because of the overall aging of the population and the fact that cancer incidence and mortality rise exponentially in the 50 - 85 years old age groups. The increased caseload of older patients with cancer will present great challenges to all components of the health care systems. As a result, there is a great need for clinical research to identify and implement evidence-based, personalized, best practices to eliminate pain and suffering from cancer in older patients, enhance their quality of life and extend their meaningful survival. There is considerable heterogeneity among patients of the same age, so that chronologic age alone provides little information regarding an individual's tolerance to cancer treatments. Geriatric oncology is going to become a major component of oncology and geriatric practice, and therefore appropriate consideration and support to it will need to be developed in public health, institutional and educative policies around the world.

\section{References}

[1] Sarkar, A. and Shahi, U.P. (2013) Assessment of Cancer Care in Indian Elderly Cancer Patients: A Single Center Study. South Asian Journal of Cancer, 2, 202-208. http://dx.doi.org/10.4103/2278-330X.119904

[2] Steer, C.B., Marx, G.M. and Singhal, N. (2009) Cancer in Older People: A Tale of Two Disciplines. Internal Medicine Journal, 39, 771-775. http://dx.doi.org/10.1111/j.1445-5994.2009.02056.x

[3] Berger, N.A., Savvides, P. and Koroukian, S.M. (2006) Cancer in the Elderly. Transactions of the American Clinical and Climatological Association, 117, 147-156.

[4] Monfardini, S. (2004) Elderly Oncology: A New Subspecialty? Journal of Clinical Oncology, 22, 4655. http://dx.doi.org/10.1200/JCO.2004.04.186

[5] Extermann, M. (2010) Geriatric Oncology: An Overview of Progresses and Challenges. Cancer Research and Treatment, 42, 61-68. http://dx.doi.org/10.4143/crt.2010.42.2.61

[6] Hutchins, L.F., Unger, J.M. and Crowley, J.J. (2000) Patients 65 Years of Age or Older in Cancer-Treatment Trials. The New England Journal of Medicine, 342, 1531. http://dx.doi.org/10.1056/NEJM200005183422016

[7] Wildiers, H., Mauer, M. and Pallis, A. (2013) End Points and Trial Design in Geriatric Oncology Research: A Joint European Organisation for Research and Treatment of Cancer-Alliance for Clinical Trials in Oncology. Journal of Clinical Oncology, 31, 3711-3718. http://dx.doi.org/10.1200/JCO.2013.49.6125

[8] Vijaykumar, D.K., Anupama, R. and Gorasia, T.K. (2012) Geriatric Oncology: The Need for a Separate Subspecialty. Indian Journal of Medical Paediatric Oncology, 33, 134-136. 
[9] Monfardini, S., Giordano, G. and Sandri, R. (2012) Bringing Geriatrics into Oncology or Also Oncology into Geriatrics? Annals of Oncology, 23, 801. http://dx.doi.org/10.1093/annonc/mdr597

[10] Kunkler, I.H., Audisio, R. and Belkacemi, Y. (2014) Review of Current Best Practice and Priorities for Research in Radiation Oncology for Elderly Patients with Cancer: The International Society of Geriatric Oncology (SIOG) Task Force. Annals of Oncology, 25, 2134-2146. http://dx.doi.org/10.1093/annonc/mdu104

[11] Kenis, C., Schuermans, H. and Van Custem, E. (2009) Screening for a Geriatric Risk Profile in Older Cancer Patients: A Comparative Study of the Predictive Validity of Three Screening Tools. Critical Reviews in Oncology/Hematology, 72, S22. http://dx.doi.org/10.1016/S1040-8428(09)70046-0

[12] Overcash, J.A., Beckstead, J. and Moody, L. (2006) The Abbreviated Comprehensive Geriatric Assessment (aCGA) for Use in the Older Cancer Patient as a Prescreen: Scoring and Interpretation. Critical Reviews in Oncology/Hematology, 59, 205-210. http://dx.doi.org/10.1016/j.critrevonc.2006.04.003

[13] Balducci, L., Lyman, G.H., Ershler, W.B. and Extermann, M. (2004) Comprehensive Geriatric Oncology. 2nd Edition, Taylor \& Francis, London \& New York.

[14] Extermann, M., Green, T., Tiffenberg, G. and Rich, C.J. (2009) Validation of the Senior Adult Oncology Program (SAOP) 2 Screening Questionnaire. Critical Reviews in Oncology/Hematology, 69, 185.

[15] Monfardini, S., Aapro, M.S. and Bennett, J.M. (2007) Organization of the Clinical Activity of Geriatric Oncology: Report of SIOG (International Society of Geriatric Oncology) Task Force. Critical Reviews in Oncology/Hematology, 62, 62-73. http://dx.doi.org/10.1016/j.critrevonc.2006.10.003

[16] White, H.K. and Cohen, H.J. (2006) The Older Cancer Patient. Medical Clinics of North America, 90, 967-982. http://dx.doi.org/10.1016/j.mcna.2006.05.017

[17] Pal, S.K., Katheria, V. and Hurria, A. (2010) Evaluating the Older Patient with Cancer: Understanding Frailty and the Geriatric Assessment. CA: A Cancer Journal for Clinicians, 60, 120-132. http://dx.doi.org/10.3322/caac.20059

[18] Surbone, A., Kagawa-Singer, M. and Terret, C. (2007) The Illness Trajectory of Elderly Cancer Patients across Cultures: SIOG Position Paper. Annals of Oncology, 18, 633-638. http://dx.doi.org/10.1093/annonc/mdl178

[19] Anisimov, V.N. (2003) The Relationship between Aging and Carcinogenesis: A Critical Appraisal. Critical Reviews in Oncology/Hematology, 45, 277-304. http://dx.doi.org/10.1016/S1040-8428(02)00121-X

[20] Flood, K.L., Carroll, M.B. and Le, C.V. (2006) Geriatric Syndromes in Elderly Patients Admitted to an OncologyAcute Care for Elders Unit. Journal of Clinical Oncology, 24, 2298-2303. http://dx.doi.org/10.1200/JCO.2005.02.8514

[21] Koroukian, S.M., Murray, P. and Madigan, E. (2006) Comorbidity, Disability, and Geriatric Syndromes in Elderly Cancer Patients Receiving Home Health Care. Journal of Clinical Oncology, 24, 2304-2310. http://dx.doi.org/10.1200/JCO.2005.03.1567

[22] Mohile, S., Dale, W. and Hurria, A. (2012) Geriatric Oncology Research to Improve Clinical Care. Nature Reviews Clinical Oncology, 9, 571-578. http://dx.doi.org/10.1038/nrclinonc.2012.125

[23] Gupta, S.K. and Lamont, E.B. (2004) Patterns of Presentation, Diagnosis, and Treatment in Older Patients with Colon Cancer and Comorbid Dementia. Journal of the American Geriatrics Society, 52, 1681-1687. http://dx.doi.org/10.1111/j.1532-5415.2004.52461.x

[24] Balducci, L. and Ershler, W.B. (2005) Cancer and Ageing: A Nexus at Several Levels. Nature Reviews Cancer, 5, 655662. http://dx.doi.org/10.1038/nrc1675

[25] Kumar, A., Soares, H.P. and Balducci, L. (2007) Treatment Tolerance and Efficacy in Elderly Oncology: A Systematic Review of Phase III Randomized Trials Conducted by Five National Cancer Institute-Sponsored Cooperative Groups. Journal of Clinical Oncology, 25, 1272-1276. http://dx.doi.org/10.1200/JCO.2006.09.2759

[26] Caillet, P., Laurent, M. and Bastuji-Garin, S. (2014) Optimal Management of Elderly Cancer Patients: Usefulness of the Comprehensive Geriatric Assessment. Clinical Interventions in Aging, 9, 1645-1660.

[27] Extermann, M. (2003) Studies of Comprehensive Geriatric Assessment in Patients with Cancer. Cancer Control, 10, 463-468.

[28] Puts, M.T., Hardt, J. and Monette, J. (2012) Use of Geriatric Assessment for Older Adults in the Oncology Setting: A Systematic Review. Journal of the National Cancer Institute, 104, 1133-1163. http://dx.doi.org/10.1093/jnci/djs285

[29] Extermann, M., Aapro, M. and Bernabei, R. (2005) Use of Comprehensive Geriatric Assessment in Older Cancer Patients: Recommendations from the Task Force on CGA of the International Society of Geriatric Oncology (SIOG). Critical Reviews in Oncology/Hematology, 55, 241-252. http://dx.doi.org/10.1016/j.critrevonc.2005.06.003

[30] Extermann, M. (2010) Evaluation of the Senior Cancer Patient: Comprehensive Geriatric Assessment and Screening Tools for the Elderly. In: Schrijvers, D., Aapro, M., Zakotnik, B., Audisio, R., van Halteren, H. and Hurria, A., Eds., Handbook of Cancer in the Senior Patient, Informa Healthcare, New York, London, 13-21. 
http://dx.doi.org/10.3109/9781841847481.003

[31] Hitz, F., Mey, U. and Clough-Gorr, K.M. (2009) Results from a Pilot Study of a Brief Cancer-Specific Geriatric Assessment (CGA) Tool for Use in Clinical Trials in Older Cancer Patients. Critical Reviews in Oncology/Hematology, 72, S20-S21. http://dx.doi.org/10.1016/S1040-8428(09)70044-7

[32] Brighi, N., Balducci, L. and Biasco, G. (2014) Cancer in the Elderly: Is It Time for Palliative Care in Geriatric Oncology? Journal of Geriatric Oncology, 5, 197-203. http://dx.doi.org/10.1016/j.jgo.2014.01.007

[33] Yee, K.W., Pater, J.L. and Pho, L. (2003) Enrollment of Older Patients in Cancer Treatment Trials in Canada: Why Is Age a Barrier? Journal of Clinical Oncology, 21, 1618-1623. http://dx.doi.org/10.1200/JCO.2003.12.044

[34] Aapro, M.S., Köhne, C.H. and Cohen, J.H. (2005) Never Too Old. Age Should Not Be a Barrier to Enrollment in Cancer Clinical Trials? Oncology, 10, 198-204. http://dx.doi.org/10.1634/theoncologist.10-3-198

[35] Kimmick, G.G., Peterson, B.L., Kornblith, A.B., Mandelblatt, J., Johnson, J.L., Wheeler, J., et al. (2004) Improving Accrual of Older Persons to Cancer Treatment Trials: A Randomized Trial Comparing an Educational Intervention with standard information: CALGB 360001. Journal of Clinical Oncology, 23, 2201-2207.

[36] Kemeny, M.M., Peterson, B.L. and Kornblith, A.B. (2003) Barriers to Clinical Trial Participation by Older Women with Breast Cancer. Journal of Clinical Oncology, 21, 2268-2275. http://dx.doi.org/10.1200/JCO.2003.09.124

[37] Kornblith, A.B., Kemeny, M., Peterson, B.L., et al. (2002) Survey of Oncologists Perceptions of Barriers to Accrual of Older Patients with Breast Carcinoma to Clinical Trials. Cancer, 95, 989-996. http://dx.doi.org/10.1002/cncr.10792

[38] Hurria, A., Naylor, M. and Cohen, H.J. (2013) Improving the Quality of Cancer Care in an Aging Population: Recommendations from an IOM Report. The Journal of the American Medical Association, 310, 1795-1796. http://dx.doi.org/10.1001/jama.2013.280416

[39] Maggiore, R.J., Gorawara-Bhat, R. and Levine, S.K. (2014) Perceptions, Attitudes, and Experiences of Hematology/Oncology Fellows toward Incorporating Geriatrics in Their Training. Journal of Geriatric Oncology, 5, 106-115. http://dx.doi.org/10.1016/j.jgo.2013.10.003

[40] Williams, G.R., Deal, A.M. and Jolly, T.A. (2014) Feasibility of Geriatric Assessment in Community Oncology Clinics. Journal of Geriatric Oncology, 5, 245-251. http://dx.doi.org/10.1016/j.jgo.2014.03.001

[41] Kalsi, T., Payne, S. and Brodie, H. (2013) Are the UK Oncology Trainees Adequately Informed about the Needs of Older People with Cancer? British Journal of Cancer, 108, 1936-1941. http://dx.doi.org/10.1038/bjc.2013.204

[42] Puts, M.T., Santos, B. and Hardt, J. (2014) An Update on a Systematic Review of the Use of Geriatric Assessment for Older Adults in Oncology. Annals of Oncology, 25, 307-315. http://dx.doi.org/10.1093/annonc/mdt386 
Scientific Research Publishing (SCIRP) is one of the largest Open Access journal publishers. It is currently publishing more than 200 open access, online, peer-reviewed journals covering a wide range of academic disciplines. SCIRP serves the worldwide academic communities and contributes to the progress and application of science with its publication.

Other selected journals from SCIRP are listed as below. Submit your manuscript to us via either submit@scirp.org or Online Submission Portal.
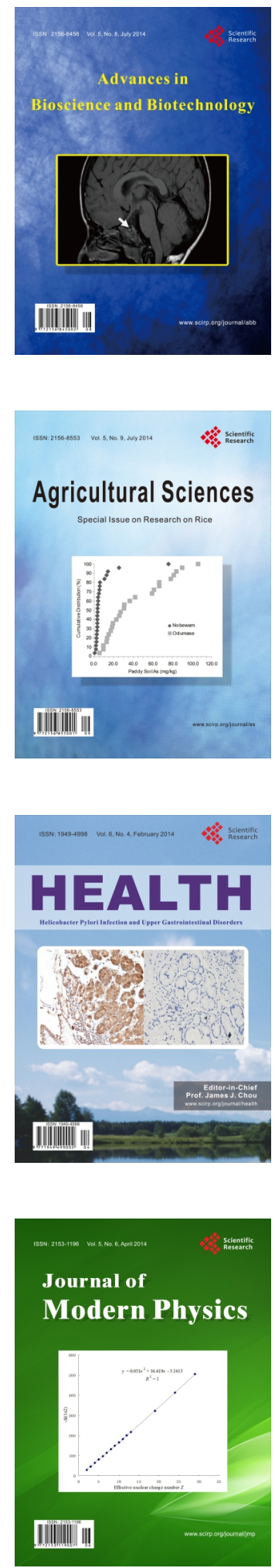
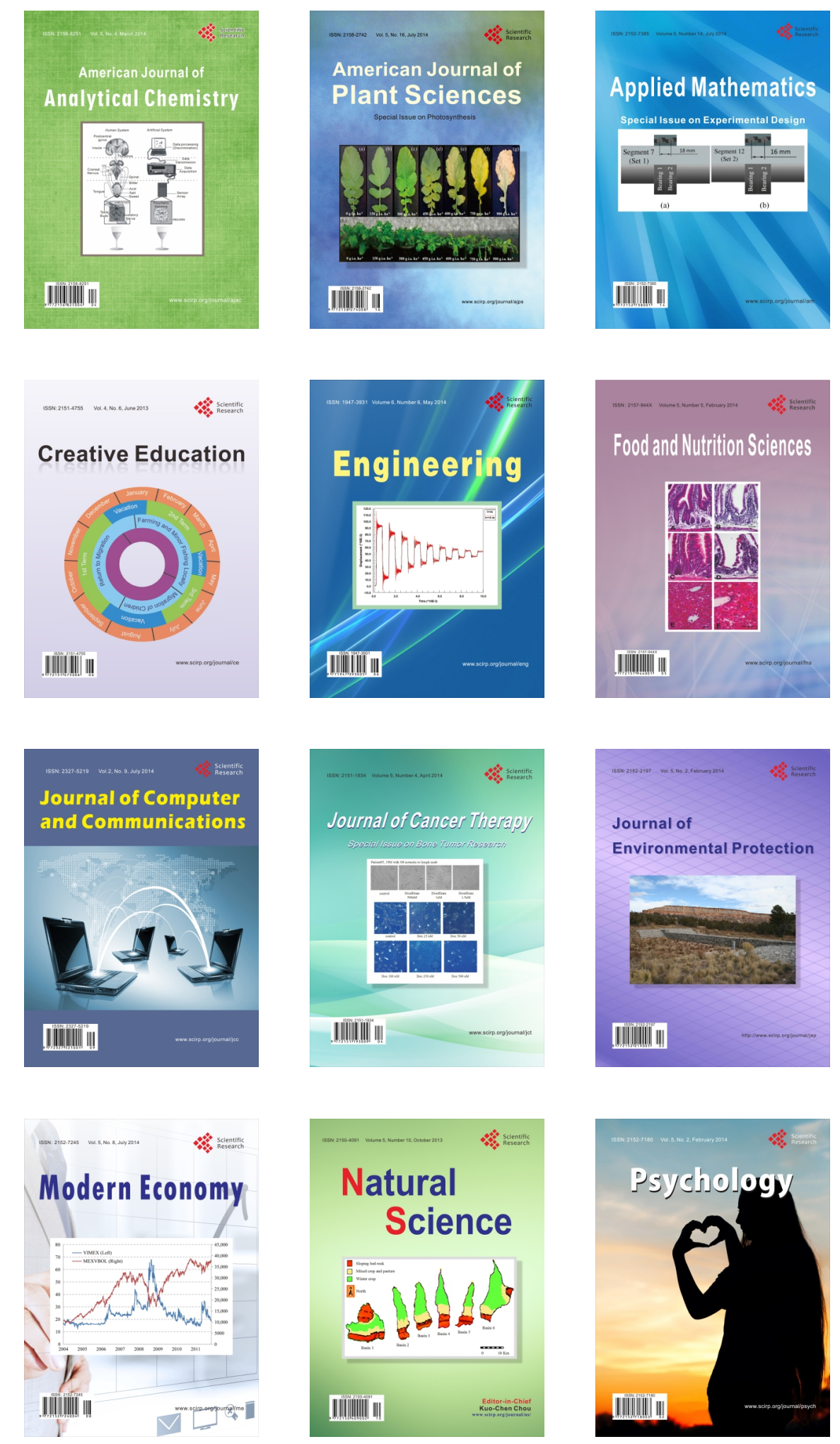